\title{
UMA ANALISE ESPACIAL DA IMIGRAÇÃO NO BRASIL
}

\author{
Karla Cristina Tyskowski Teodoro Rodrigues \\ Mestranda em Economia Regional da Universidade Estadual de Londrina (UEL). \\ Endereço para contato: Rua Benedito Salles, 312 - Carlópolis - PR \\ CEP: 86420-000 - E-mail: karlatyskowski@gmail.com
}

\author{
Marcos Aurélio Brambilla \\ Mestre em Economia Regional pela Universidade Estadual de Londrina (UEL). \\ Endereço para contato: Avenida Santo Berloffa, 63 - Centro - Paiçandu - PR \\ CEP: 87140-000 - E-mail: marcos-brambilla@hotmail.com
}

\section{Marcia Regina Gabardo da Camara}

Professor Associada do Departamento de Economia da Universidade Estadual de Londrina (UEL).

Endereço para contato: Rua Belo horizonte, 734 - Londrina - PR

CEP: 86020-060 - E-mail: mgabardo@uel.br

\section{Auberth Henrik Venson \\ Mestrando em Economia Regional da Universidade Estadual de Londrina (UEL). Endereço para contato: Rua Professor João Candido, 1404 - Centro - Londrina - PR \\ CEP: 86010-001 - E-mail: auberth.eco@gmail.com}

Recebido em 09 de junho de 2015. Aceito em 25 de agosto de 2015.

\section{RESUMO}

O objetivo do trabalho é verificar a existência de relação espacial entre a imigração e rendimento médio no Brasil para o ano de 2010. Utiliza-se a metodologia de Análise Exploratória de Dados Espaciais (AEDE). Foi realizada a análise univariada da imigração e a análise bivariada da imigração e rendimento médio para identificar os coeficientes bivariados I de Moran, os gráficos de dispersão, os mapas de significâncias e os mapas de clusters das variáveis dos municípios. Os dados foram retirados do Instituto Brasileiro de Geografia e Estatística (IBGE). A análise dos resultados da AEDE permite verificar que a concentração de municípios com os maiores valores de imigração está nas regiões Centro Oeste e Sul do Brasil, e a concentração de municípios com os menores valores de imigração estão no Norte, Nordeste e Sudeste do país e no Rio Grande do Sul. Verifica-se a presença de relação espacial positiva entre rendimentos médios superiores e a imigração no ano de 2010.

\section{Palavras-chave}

Imigração, AEDE, População.

\begin{abstract}
The objective of the research is to verify through analysis Exploratory Spatial Data (ESDA) spatial existence between immigration and average income in Brazil for the year 2010. Univariate analysis of immigration and the bivariate analysis of immigration and middleincome were performed. We calculated the bivariate coefficients Moran's I, scatter plots, the significance of maps and maps of clusters of variables municipalities. Data were taken from the Brazilian Institute of Geography and Statistics (IBGE). The ESDA results revealed the concentration of municipalities with the highest immigration figures are in middle western and southern Brazil, and the concentration of municipalities with the lowest migration values are in the North, Northeast and Southeast of the country and Rio Grande do Sul. We verified
\end{abstract}


the positive spatial relation between immigration and middle wage in migration processes during the year of 2010 .

\section{Keywords}

Migration, ESDA, Population.

JEL CLASSIFICATION: C38, J10, R1

\section{Introdução}

O Brasil é um país de tamanho continental e de várias etnias, cuja característica marcante é a imigração de trabalhadores entre as suas regiões. Estudiosos da população e da desigualdade da renda, como Langoni (1973), associam as disparidades econômicas regionais e a imigração interna, especialmente aquela motivada pelo mercado de trabalho.

O estudo dos movimentos migratórios tem papel relevante na literatura econômica, principalmente no que se refere aos fatores que motivam as pessoas a se deslocarem de seu local de origem para outro país ou região, ao perfil do migrante e ao impacto desses fluxos sobre a desigualdade de renda entre países ou entre regiões de um mesmo país (TONET e HERMETO, 2011).

Greenwood e Hunt (2003) relatam que do ponto de vista metodológico, até 1960 os trabalhos sobre imigração eram descritivos e a partir de então começaram a ganhar formalização e utilização de modelos empíricos. Os autores expõem que do ponto de vista teórico estudos que respaldam o fenômeno da imigração em teorias de equilíbrio e outros que se baseiam em teorias de desequilíbrio. Ainda discutindo as diversas nuances do estudo de imigração, os autores enfatizam a discussão sobre o nível de agregação escolhido pelos pesquisadores deste campo de estudo.

Os trabalhos de Sachsida et al. (2009), Justo e Silveira Neto (2008), Tonet e Hermeto (2011), Sjaastad (1962) e Mata (1973) ressaltam as diferentes motivações para a migrar, como investimento em capital humano e perspectivas melhor condição social. Assim como no contexto migratório, o aumento das migrações interestaduais e inter-regionais é o tema de estudo de Feguglia e Procópio (2013), Camara (1988), Azzoni, Carmo e Menezes (2003), entre outros. Estudos mostram que o diferencial salarial não ocorre apenas em função do nível de escolaridade, mas também por meio de segregação, onde o gênero e a raça fazem com que se observem assimetrias e desigualdades nos rendimentos dos indivíduos.

Diante disso, o presente artigo tem por objetivo preencher uma lacuna que é analisar as relações espaciais entre o rendimento médio e a imigração interna no Brasil em 2010, a análise é feito por meio da Analise Exploratória de Dados Espaciais (AEDE) com o intuito de verificar existência de dependência espacial entre a imigração e o rendimento médio no Brasil para ano de 2010, foi realizada a análise univariada da imigração e a análise bivariada da imigração e rendimento médio.

Este artigo está organizado em cinco seções: introdução, revisão de literatura que descreve as características da população que migratória, metodologia, análise de resultados e considerações finais. 


\section{Condição de imigração: teorias e evidências empíricas}

As teorias sobre imigração em suas origens buscaram descrever o processo de desenvolvimento como um todo e neste contexto inseriram os movimentos migratórios populacionais, focando a passagem de sociedades tradicionais para sociedades modernas na Europa (CAMARA, 1988). As análises econômicas supunham que o processo de desenvolvimento econômico ocorria em condições de oferta ilimitada de mão-de-obra pela teoria. Camara (1988) e Brito (2009) afirmam que as discussões econômicas e sociológicas sobre o tema também foram influenciadas pela teoria da modernização social. Os autores destacam que o primeiro conjunto de teorias encontra subsídios para análise dos movimentos migratórios, enquanto o segundo conjunto de estudos busca referências na análise históricoestrutural. A teoria neoclássica destaca os fatores de atração dos migrantes para as cidades, tendo em vista a experiência de desenvolvimento dos países europeus, enquanto a teoria histórico estrutural focaliza os fatores de expulsão dos migrantes, a partir da experiência dos países em desenvolvimento. Adicionalmente, Fausto Brito (2007, p.3) afirma que nas teorias neoclássicas:

\footnotetext{
Mesmo que ocorram obstáculos intervenientes, associados à distância ou aos custos de transportes, assim como aos problemas psicossociais inerentes ao translado, a virtuosidade econômica e social das migrações é um pressuposto inegável dessas teorias. Essa dimensão positiva das migrações, tanto do ponto de vista social, quanto econômico, serve de pano de fundo para a racionalidade da decisão de emigrar. A análise custo-benefício, em todas as suas dimensões, tende a ser amplamente favorável aos benefícios. Emigrar em direção às grandes regiões urbanas é a opção mais adequada quando o objetivo é a melhoria do padrão de vida, mesmo quando se considera a enorme adversidade que essas regiões impõem aos imigrantes e suas famílias.
}

Muitos são os estudos que buscam explicitar as motivações econômicas e características da população imigrante, bem como nos diferenciais salariais decorrentes da imigração. Segundo Taylor (1999), as origens e impactos da imigração não devem ser estudados separadamente nem de forma isolada em relação ao processo de desenvolvimento, pois isso gera limitações para a análise. $\mathrm{O}$ autor expõe que o nível de desenvolvimento de uma região influencia as decisões de migrar e estas, por sua vez, têm impactos sobre o desenvolvimento, especialmente nos países de origem dos migrantes.

Ravenstein (1885) foi o precursor dos estudos sobre imigração. Ele enumerou as suas leis sobre a imigração, que foram aprimoradas por outros teóricos, e representaram um primeiro passo na tentativa de se compreender os elementos motivadores da imigração. $\mathrm{O}$ autor relatou que a imigração partia de pressupostos, que identificavam os movimentos migratórios relacionados com o desenvolvimento do capitalismo; a população e as atividades econômicas estão espacialmente distribuídas de forma desigual, havendo excedente de mão de obra em algumas regiões e escassez em outras; movimentos migratórios a procura emprego e deslocamento em buscando melhoria em sua situação material.

A percepção de que as decisões de imigração são motivadas principalmente pelos diferenciais de salários é o ponto de partida de praticamente todos os estudos modernos sobre as decisões de imigração que analisam a mobilidade de trabalhadores como um investimento em capital humano (BORJAS, 1989). 
A imigração, contém dois aspectos importantes: a racionalidade dos indivíduos e o fato de a decisão de migrar ser um fenômeno que envolve a decisão individual de agente, mas sim de um conjunto de indivíduos ou uma família. O primeiro aspecto, conforme destacam os teóricos da microeconomia neoclássica, envolve a hipótese de que os indivíduos são racionais e capazes de ordenar suas preferências, com base em cálculos racionais, visando a maximizar a utilidade de suas escolhas, tendo em vista maiores chances de inserção social a partir das informações do mercado de trabalho da origem e destino dos migrantes (DURHAM, 1973). Segundo Borjas (1989), a ideia central destas discussões seria a de políticas de imigração com o objetivo que captar capital humano e físico da massa migratória, assim, níveis na atividade econômica, afetariam os fluxos migratórios.

O segundo aspecto, Santos et al. (2010) fala que na economia contemporânea de imigração de trabalhadores, é o de que a decisão de migrar não é tomada por indivíduos isolados, mas por um conjunto maior de pessoas que estão de alguma forma ligados, pois assim se sentem intuitivamente mais seguros. Taylor (1986) ressalta que os agentes não visam apenas o lucro, mas também a minimização dos riscos. Para Singer (1973) as migrações são historicamente condicionadas, sendo o resultado de um processo global de mudança, do qual elas não devem ser separadas. O processo de imigração está diretamente associado com o desenvolvimento do capitalismo, principalmente com o processo de industrialização provocado por este.

Tonet e Hermeto (2011) ressaltam que embora uma variedade de modelos teóricos tenha sido desenvolvida para explicar a imigração internacional, a literatura sobre imigração interna tem se expandido rapidamente e ganhado maior destaque nas últimas décadas. A imigração interna é um fenômeno significativo no Brasil desde o século $\mathrm{XX}$, em que a população migrava em busca de postos de trabalho no Sudoeste. A abordagem de questões relacionadas a esse fenômeno, como a identificação de possíveis fatores determinantes desses movimentos migratórios e do perfil do migrante interno, tem se destacado na literatura nacional.

Desta forma, de acordo com a teoria global de Lee (1966), a decisão de migrar responde à "pressão" da pobreza no lugar de origem, à "atração" da perspectiva de obter rendas elevadas no lugar de destino, assim como à intervenção de outros obstáculos e fatores pessoais.

Paralelamente à diversidade dos mercados de trabalho regionais, tem-se que a motivação do deslocamento também é bastante distinta entre os sexos. Oliveira e Jannuzzi (2004) discorrem que a procura por emprego corresponde à motivação predominante para imigração dos homens, no caso das mulheres, tem se como relevância principal a necessidade de acompanhar a família. Esta diferenciação não só interfere na decisão de trabalhar uma vez instalada no local de destino, como também influencia na magnitude do salário reserva de homens e mulheres migrantes.

Camara (1988) discorre sobre as características da população que migra, as circunstâncias do deslocamento, a absorção dos migrantes dentro do marco social e cultural da nova sociedade. Adquirem importância no enfoque modernizante o ritmo e a sequência do processo de transformação. Existe também um importante aspecto da imigração que se refere à realocação de empregos no mercado de trabalho, como resultado de choques econômicos. Tornou-se uma particularidade comum das economias de mercado cuja flexibilidade 
constitui-se frequentemente num aspecto positivo da economia, permitindo que a pessoa procure um local que atenda suas necessidades da melhor forma. A preocupação cada vez maior com problemas de crescimento econômico e melhor distribuição de renda, fez com que a análise do mercado de trabalho, fosse cada vez mais valorizada. Segundo Lima (1980), no Brasil o interesse iniciou-se, na década de 1960, com a teoria do capital humano. O autor salienta que a melhoria no nível de especialização dos trabalhadores, o aumento das habilitações, de proporção crescente da população e maior acumulação de conhecimento são conhecidos como fatores importantes para crescimento econômico.

Segundo Mincer (1958), a teoria do capital humano é a mais utilizada para justificar diferenças salariais persistentes no mercado de trabalho, mesmo quando este se encontra em equilíbrio, partindo do princípio de que a remuneração de trabalhador é determinada de acordo com o investimento realizado pelo mesmo em educação e treinamento. A percepção de que as decisões de imigração são motivadas principalmente pelos diferenciais de salários é o ponto de partida de praticamente todos os estudos modernos sobre as decisões de imigração que analisam a mobilidade de trabalhadores como um investimento em capital humano (BORJAS, 1989).

Posteriormente, surgiram trabalhos sobre a teoria do mercado dual (segmentação) onde existe o mercado primário e secundário, o primeiro se caracteriza por empregos estáveis, salario relativamente altos, produtividade alta, com maior qualificação dos trabalhadores. Já o mercado secundário é caracterizado por alta rotatividade dos trabalhadores, baixa produtividade, baixa qualificação e níveis altos de desemprego (LIMA, 1980).

Sjaastad (1962) enfoca o problema da imigração como um investimento em capital humano, de acordo com sua teoria, o indivíduo comporta-se como se comparasse os custos e os retornos da imigração, agindo com base em tal comparação. Como principais desembolsos diretos, que são consequentes à imigração, deveriam ser considerados os gastos com alimentação, alojamento e transporte. Entre os custos indiretos, considerar-se-iam as receitas que deixam de ser auferidas durante a viagem e o tempo com a procura de emprego e a aquisição do treinamento correspondente; tais custos seriam função da distância entre os locais de origem e de destino e da taxa de desemprego prevalecente neste último.

Segundo Mata (1973), as migrações internas no Brasil começaram a desempenhar um papel de destaque na recomposição espacial da população, somente a partir da década de 1930. Acompanhando a evolução das atividades produtivas, a população foi deslocando-se do campo para as cidades; entre 1940 e 1970, por exemplo, a população rural cresceu à taxa média anual de $1,8 \%$, enquanto a urbana expandiu-se 4,8\%. Em seu artigo, o autor dá ênfase ao efeito mais visível das migrações internas, que segundo esse, seria a crescente concentração da população em aglomerados urbanos.

Nos trabalhos empíricos que utilizam os dados da Pesquisa Nacional por Amostra de Domicílio (PNAD) para fazer a análise dos movimentos migratório no país, destacam-se Sachsida et al. (2009), os autores utilizam os dados de 2006 para definir o perfil do migrante brasileiro, concluindo que as variáveis idade e nível educacional afetam a decisão de migrar e, desta forma, os jovens com alto nível de escolaridade são os indivíduos mais propensos a migrar. Por sua vez, Justo e Silveira Neto (2008) fornecem evidências a respeito do migrante interno a partir dos microdados dos Censos Demográficos de 1980, 1991 e 2000, expondo que os migrantes, além de jovens e mais escolarizados em relação ao lugar de onde migraram, 
veem com maior probabilidade de Unidades da Federação (UF) em condições sociais relativamente mais precárias.

O estudo de Golgher et al. (2005) verifica os determinantes da imigração entre as mesorregiões brasileiras, e conclui que a interação entre as características regionais e os aspectos individuais tem influência sobre a decisão de migrar. Sachsida et al. (2010) analisam o efeito da distribuição de renda e das transferências federais para as Unidades da Federação (UF's) sobre o fluxo de migrantes recebido por estas; os autores concluem que as transferências federais têm efeito positivo sobre a imigração, e que a concentração de renda desestimula a imigração, o que indica que os migrantes buscam UF's com distribuição de renda mais equitativa.

Nesse contexto, Azzoni, Carmo e Menezes (2003) analisam o diferencial salarial entre as regiões metropolitanas (RMs) do Brasil, buscando identificar se, além do capital humano, o diferencial de custo de vida e as características regionais também possuem um papel na sua explicação. Eles verificam que o diferencial de custo de vida, embora relevante, não é capaz, individualmente, de explicar os diferenciais salariais. O Censo do IBGE 2010 aponta que um dos os maiores problemas do nosso país é a concentração de renda, onde existem poucas pessoas ganhando muito dinheiro e muitas pessoas ganhando pouco dinheiro. $\mathrm{O}$ estudo confirma essa situação, visto que em seus resultados $46,34 \%$ das pessoas com 10 anos ou mais de idade ganhavam até 2 salários mínimos, enquanto que o percentual de pessoas de 10 anos ou mais de idade, que recebiam de 5 salários-mínimos ou mais, era apenas de 5,26\%.

Os custos da imigração podem ser elevados e, inicialmente, o migrante pode receber menor remuneração que o não migrante. Entretanto, existe um processo de assimilação do trabalhador, após a imigração, no qual a evolução de seu salário é maior que a do não migrante, podendo possibilitar vantagem de migrar no longo prazo (FREGUGLIA; PROCOPIO, 2013).

Mata (1973), preocupou-se com a concentração populacional nos centros urbanos e constatou que o processo de desenvolvimento mantido pelo governo, corroborou para o crescimento produtivo e assim para o incentivo de crescimento populacional da região, podendo desta forma estabelecer concentração ou desconcentração da população e da região.

Costa (1975) analisou as vantagens e desvantagens dos migrantes em relação aos não migrantes no meio urbano. As vantagens constatadas por ele foram significantes, tratando-se de maior facilidade nos diversos ramos de atividade econômica e oferecendo mobilidade vertical superior a dos não migrantes, ratificada pelo número maior de empregadores e pela renda maior nas regiões analisadas.

\subsection{Imigração e distribuição de renda}

A revisão de literatura permitiu verificar que a imigração muitas vezes tem como motivação a melhoria de rendimento e a condição social do migrante. Desta forma, Sachsida et al. (2010) fazem uma análise em dados em painel da distribuição de renda, transferências federais e imigração para as unidades da federação do Brasil, e verificam a influência dos fluxos migratórios sobre variáveis como violência, oportunidades econômicas, distribuição de renda, distância e transferências federais relativas ao Fundo de Participação dos Estados 
(FPE) e ao Fundo de Participação dos Municípios (FPM), deles salientam a importância de estudar estas informações agregadas.

De acordo com Golgher, Rosa e Araújo Junior (2005), há forte correlação entre a imigração e processo de desenvolvimento econômico de determinada região, de forma que quanto mais intenso de desenvolvimento, maior será a atração de imigrantes. O fenômeno migratório no Brasil foi intenso na segunda metade do sec. XX, em função da maior mobilidade e da globalização, fatores que facilitaram a imigração interna. Nas décadas de 1960 e 1970, o principal fluxo migratório era dos estados da região Nordeste para São Paulo e Rio de Janeiro. Na década de 1980, fluxo migratório importante ocorreu dos estados do Sul em direção às regiões agrícolas do Centro-Oeste e Norte, graças à grande disponibilidade de terras.

Sachsida et al. (2010) expõe que, na década de 1990, ocorreram mudanças no fluxo migratório, devido a pelo menos três fatores: i) desenvolvimento econômico das grandes metrópoles nordestinas; ii) políticas públicas adotadas pelo governo; e iii) dificuldade de empregos e crescimento da violência nos grandes centros do país. $\mathrm{O}$ autor salienta que existe uma longa tradição de atribuir a imigração ao diferencial de renda entre regiões, em virtude do qual os indivíduos e famílias migrariam das localidades mais pobres para as mais ricas. Mas, existe pouca discussão sobre a distribuição de renda resultante deste fenômeno. Em principio, a imigração de pessoas qualificadas parece indicar que a distribuição de renda melhora dentro das localidades, mas piora entre elas, pois a concentração geraria regiões com alto ou baixo capital humano o que aumentaria a desigualdade entre as regiões. Mas esta análise intuitiva é incompleta, pois não leva em consideração as interações entre trabalho qualificado e não qualificado.

Após 1960, a literatura sobre imigração interna se expande no Brasil e foram feitos importantes no estudo da imigração interna; os pioneiros no assunto são os estudos de Graham e Holanda Filho (1973), e Mata, Carvalho e Silva (1973). A partir de 2000, é renovado o interesse de pesquisadores sobre o tema, utilizando novas metodologias e bases de dados diferenciadas, e emergem novos estudos sobre o tema migrações internas no Brasil. Entre as pesquisas recentes, destaca-se o artigo de Golgher, Rosa e Araújo Junior (2005) averigua os determinantes da imigração entre as mesorregiões brasileiras. De modo geral, o artigo demonstra que o tamanho da população, tanto da região de origem quanto da região de destino, e a distância entre as regiões são determinantes significativos da imigração. Menezes e Ferreira Jr. (2003), verificam o impacto da imigração sobre a velocidade de convergência dos estados brasileiros. Os resultados econométricos dos autores confirmam a teoria da aglomeração: as migrações têm como destino as grandes cidades, onde a renda esperada é mais elevada e a densidade demográfica é maior.

Souchad e Fusco (2012, p.15) analisam os movimentos migratórios no território brasileiro, do século XIX até 2000, e verificam que as migrações internacionais e internas contribuíram para a um "movimento de redistribuição da população (de fronteira, êxodo rural, mobilidade intraurbana centro/periferia), bem como a integração e produção do espaço (colonização agrária, periferia urbana). Na atualidade, os impactos territoriais da imigração ocorrem mais em áreas já consolidadas, resultando em crescimento populacional baixo ou moderado, onde a demanda e a possibilidade de inserção são menos diversificadas. 


\section{Procedimentos Metodológicos}

Nesta seção é abordada a metodologia utilizada na pesquisa, primeiramente é apresentada a descrição das variáveis utilizadas e os correspondentes bancos de dados e em seguida é detalhada a metodologia de análise exploratória de dados espaciais.

\subsection{Descrição das variáveis e bases de dados}

Para realização do presente estudo foram empregados dados referentes à imigração e rendimento médio dos municípios brasileiros. A base de dados utilizada é proveniente do Censo Demográfico do ano de 2010, realizado e disponibilizado pelo Instituto Brasileiro de Geografia e Estatística (IBGE).

Para mensurar a imigração utilizou-se a divisão simples dos nascidos no estado pela população total para cada município em 2010, multiplicando por 100. Para a variável, rendimento médio, utilizou-se a própria variável do Censo, no qual representa a média dos rendimentos de todos os trabalhos das pessoas ocupadas de 18 anos ou mais de idade.

As variáveis então são definidas como sendo:

$$
\begin{gathered}
\qquad \text { Indice de imigração }=\frac{\text { habitantes do município nascidos no estado }}{\text { Poptulação total do município }} \times 100 \\
\text { Rendimento médio municipal }=\frac{\text { Rend. do trabalho de todas as pessoas ocupados acima de } 18 \text { anos do município }}{\text { População total do município }}
\end{gathered}
$$

A análise dos dados foi realizada com a utilização do software GeoDa.

\subsection{Análise Exploratória de Dados Espaciais (AEDE)}

A análise exploratória de dados espaciais é a técnica utilizada para descrever e visualizar distribuições espaciais, identificar localidades espaciais atípicas, descobrir padrões de associação espacial e sugerir diferentes regimes espaciais. O primeiro passo no estudo de AEDE é testar a hipótese de que os dados espaciais sejam distribuídos aleatoriamente. Intuitivamente, aleatoriedade espacial significa que os valores de um atributo numa região não dependem dos valores deste atributo nas regiões vizinhas (ALMEIDA, 2012).

No entanto essa análise é mais apropriada na investigação de variáveis espacialmente densas ou intensivas, ou seja, variáveis que são divididas por algum indicador de intensidade (per capita, etc.). Nesse sentido, Perobelli et al. (2007) reforçam que, a partir da AEDE, é possível extrair medidas de autocorrelação espacial global e local, investigando a influência dos efeitos espaciais por intermédio de métodos quantitativos.

\subsubsection{Matrizes de Pesos Espaciais}

Econ. e Desenv., Santa Maria, vol. 27, n.1, p. 164 - 182, jan. - jul. 2015 
De acordo com Almeida (2012), o conceito de matrizes de pesos espaciais é baseado na proximidade, que, por sua vez, pode ser definida de acordo com a vizinhança, a distância tanto geográfica quanto socioeconômica, bem como um a combinação disso. Vários resultados, em econometria espacial, são sensíveis à escolha da matriz de pesos espaciais. Portanto, a discussão a respeito da tipologia das matrizes é questão importante e delicada na literatura. Das formas de matrizes de pesos espaciais utilizadas, as mais comuns são a convenção rainha e torre apresentada na figura 1.

Figura 1 - Matrizes de contiguidade

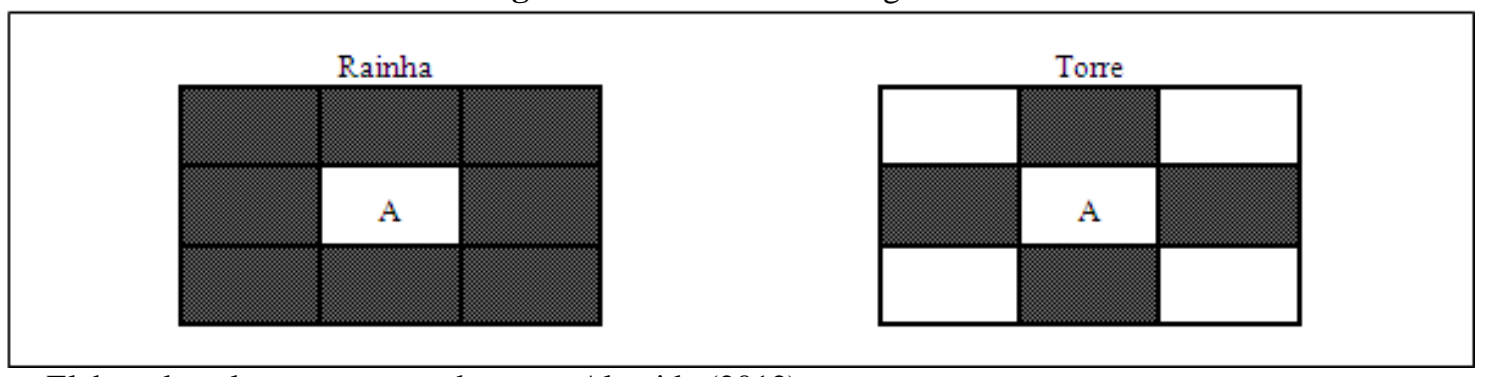

Fonte: Elaborado pelos autores com base em Almeida (2012)

A convenção de contiguidade é dita ser rainha, caso além das fronteiras com extensão diferente de zero, puderem ser considerados os vértices (nós), na visualização de um mapa, como contíguos, ou seja, no caso das variáveis em estudo imigração e rendimento médio, essa convenção considera a relação entre as variáveis não só das fronteiras com extensão diferente de zero, mas os vértices também, e a origem do nome se deve ao movimento da peça de xadrez rainha, no qual se desloca no tabuleiro em extensões diferente de zero e pelos vértices (diagonal). Caso apenas as fronteiras físicas com extensão diferente de zero entre as regiões sejam levadas em conta, a convenção de contiguidade é considerada como torre, essa convenção se diferencia da convenção rainha, apenas por não considerar a relação das variáveis nos vértices, e o nome se origina do movimento da peça de xadrez torre, em que se difere da rainha por não se deslocar no tabuleiro pelos vértices (diagonal). Essas duas convenções são as mais utilizadas na literatura (ALMEIDA, 2012).

\subsubsection{A Estatística I de Moran}

A estatística I de Moran é utilizada para verificar a existência de autocorrelação espacial de uma variável em uma determinada região. Foi proposta por Patrick A. P. Moran no ano de 1948. Algebricamente a estatística I de Moran é representada pela equação 1:

$$
\mathrm{I}=\frac{\mathrm{n}}{\mathrm{S}_{0}} \frac{\sum_{\mathrm{i}} \sum_{\mathrm{j}} \mathrm{w}_{\mathrm{ij}} \mathrm{z}_{\mathrm{i}} \mathrm{z}_{\mathrm{j}}}{\sum_{\mathrm{i}=1}^{\mathrm{n}} \mathrm{z}^{2}}
$$

Ou matricialmente, a equação 1 é representada pela equação 2 :

$$
\mathrm{I}=\frac{\mathrm{n}}{\mathrm{S}_{0}} \frac{\mathrm{z} \prime \mathrm{Wz}}{\mathrm{z} \prime \mathrm{z}}
$$


Em que $n$ é o número de regiões, $z$ denota os valores da variável de interesse padronizada, $W z$ representa os valores médios da variável de interesse padronizada nos vizinhos, definidos segundo uma matriz de ponderação espacial $W$. Um elemento dessa matriz, referente à região $i$ e a região $j$, é registrado como $w_{i j}$, significando que todos os elementos da matriz de pesos espaciais $w$ devem ser somados.

Os valores de $i$ maiores (ou menores) do que o valor esperado de $\mathrm{E}(\mathrm{I})=[-1 /(\mathrm{n}-1)]$ indicam que há autocorrelação positiva (ou negativa). De acordo com Almeida (2012), a autocorrelação espacial positiva revela que existe uma similaridade entre os valores do atributo estudado e da localização espacial do atributo. A autocorrelação espacial negativa revela, por sua vez, que existe uma dissimilaridade entre os valores do atributo considerado e a localização espacial.

Segundo Almeida (2012), o I de Moran fornece três tipos de informações: 1) o nível de significância fornece a informação sobre os dados estarem distribuídos aleatoriamente ou não; 2) o sinal positivo da estatística I de Moran, desde que significativo, indica que os dados estão concentrados em regiões; o sinal negativo, por sua vez, indica a dispersão dos dados; 3) a magnitude da estatística fornece a força da autocorrelação espacial, quanto mais próximo de 1 mais forte é autocorrelação e quanto mais próximo de -1 mais disperso estão os dados.

\subsubsection{I de Moran Global Bivariado}

A autocorrelação espacial global bivariada permite verificar se uma variável observada em determinada região tem alguma associação com outra variável em regiões vizinhas. $\mathrm{O}$ coeficiente I de Moran bivariado é dado pela equação abaixo:

$$
\mathrm{I}^{\mathrm{Z}_{1} \mathrm{Z}_{2}}=\frac{\mathrm{n}}{\mathrm{S}_{0}} \frac{\mathrm{Z}_{1}^{\prime} W \mathrm{Z}_{2}}{\mathrm{Z}_{1}^{\prime} \mathrm{Z}_{1}}
$$

Onde $\mathrm{WZ}_{2}$ representa a defasagem da variável $\mathrm{Z}_{2}$. A presença de autocorrelação espacial positiva indica uma associação dos valores das variáveis que está sendo estudada e de suas localizações. Sendo assim, a autocorrelação positiva mostra que municípios com uma alta (baixa) imigração são rodeados por municípios com um alto (baixo) rendimento médio.

O I de Moran esperado é dado por E $(\mathrm{I})=[-1 /(\mathrm{n}-1)]$, fornece o valor que seria obtido se não houvesse padrão espacial nos dados. Sendo que os valores de I de Moran acima da esperança de Moran indicam autocorrelação espacial positiva e os valores abaixo indicam autocorrelação negativa (ROCHA e PARRÉ, 2008).

\subsubsection{O Diagrama de Dispersão de Moran}

Segundo Almeida (2012), o diagrama de dispersão de Moran é uma alternativa para visualizar a autocorrelação espacial, o qual mostra a defasagem espacial da variável de interesse no eixo vertical e o valor da variável de interesse no eixo horizontal. Além da medida global de associação linear espacial, o diagrama dispersão mostra associação espacial entre as regiões e seus vizinhos dividido em quatro quadrantes: alto-alto (AA), baixo-baixo (BB), alto-baixo (AB) e baixo-alto (BA), como mostra a Figura 2. 
Um agrupamento alto-alto (AA) significa que as unidades espaciais pertencentes a esse agrupamento exibem valores altos da variável de interesse (A) rodeados por unidades espaciais que apresentam valores também altos da variável defasada (B), representado pelo primeiro quadrante do diagrama. Um agrupamento baixo-alto (BA) concerne a um cluster no qual uma unidade espacial qualquer com um baixo valor da variável de interesse (A) são circundados por unidades espaciais com alto valor da variável (B). Isso é representado no segundo quadrante.

Figura 2 - Diagrama de dispersão de Moran

Autocorrelação Positiva

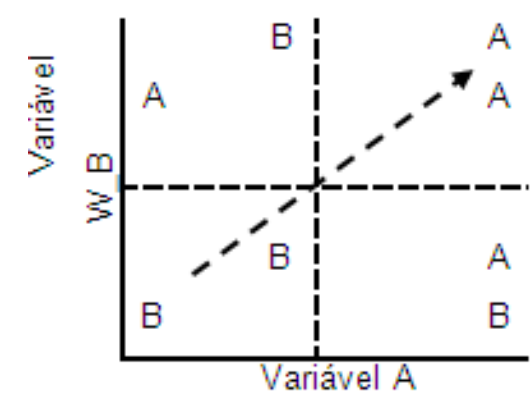

Fonte: Elaborado pelos autores com base em Almeida (2012)

Autocorrelação Negativa

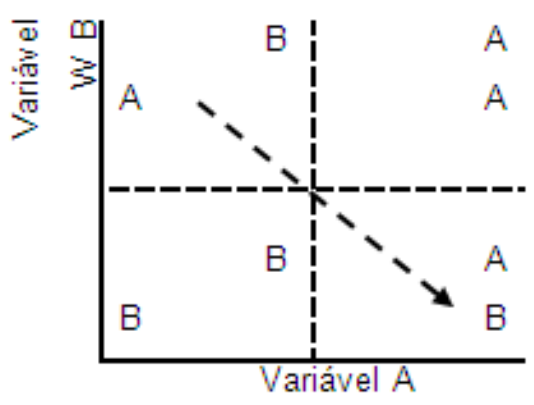

A

Um agrupamento baixo-baixo (BB) refere-se a um agrupamento cujas unidades espaciais mostram valores baixos da variável (A) circundados por unidades espaciais que ostentam valores também baixos da defasagem da variável (B), representado pelo terceiro quadrante. Um agrupamento alto-baixo $(\mathrm{AB})$ diz respeito a um cluster no qual uma unidade espacial qualquer com um alto valor da variável de interesse (A) são vizinhos de unidades espaciais com um baixo valor da variável (B). Isso é representado pelo quarto quadrante.

\subsubsection{Análise Bivariada Local}

Segundo Almeida (2012), assim como se pôde obter um coeficiente de autocorrelação espacial global num contexto bivariado, também é possível conseguir uma medida de autocorrelação espacial local multivariada. Readaptando o I de Moran local obtemos a equação 4:

$$
\mathrm{I}_{\mathrm{i}}^{\mathrm{Z}_{1} \mathrm{Z}_{2}}=\mathrm{Z}_{1 \mathrm{i}} \mathrm{WZ}_{2 \mathrm{i}}
$$

Em que $\mathrm{WZ}_{2 \mathrm{i}}$ é a defasagem espacial da variável padronizada $\mathrm{z}_{2 \mathrm{i}}$. Semelhantemente à fórmula do calculo do I de Moran na equação 3, somente os vizinhos da observação $i$, definidos conforme uma matriz de pesos espaciais, são incluídos no cálculo.

De acordo com Anselin et al. (2003, apud Almeida, 2012), essa estatística dá uma indicação do grau de associação linear (positiva ou negativa) entre o valor para uma variável em uma dada locação $i$ e a média de uma outra variável nas locações vizinhas. É possível mapear os valores da probabilidade da medida, estatisticamente significativos, gerando o chamado mapa de significância bivariado do Moran local. 


\section{Análise dos resultados}

Nesta seção são apresentados os resultados encontrados da análise exploratória de dados espaciais das seguintes variáveis: imigração e rendimento médio nos municípios brasileiros no ano de 2010.

Na figura 1, é apresentado o mapa de desvio padrão da taxa de imigração dos municípios brasileiros. Foram observados os resultados com um e dois desvios padrão abaixo e acima da média, além dos outliers abaixo e acima da média.

Figura 1 - Mapa de desvio padrão da Imigração de 2010

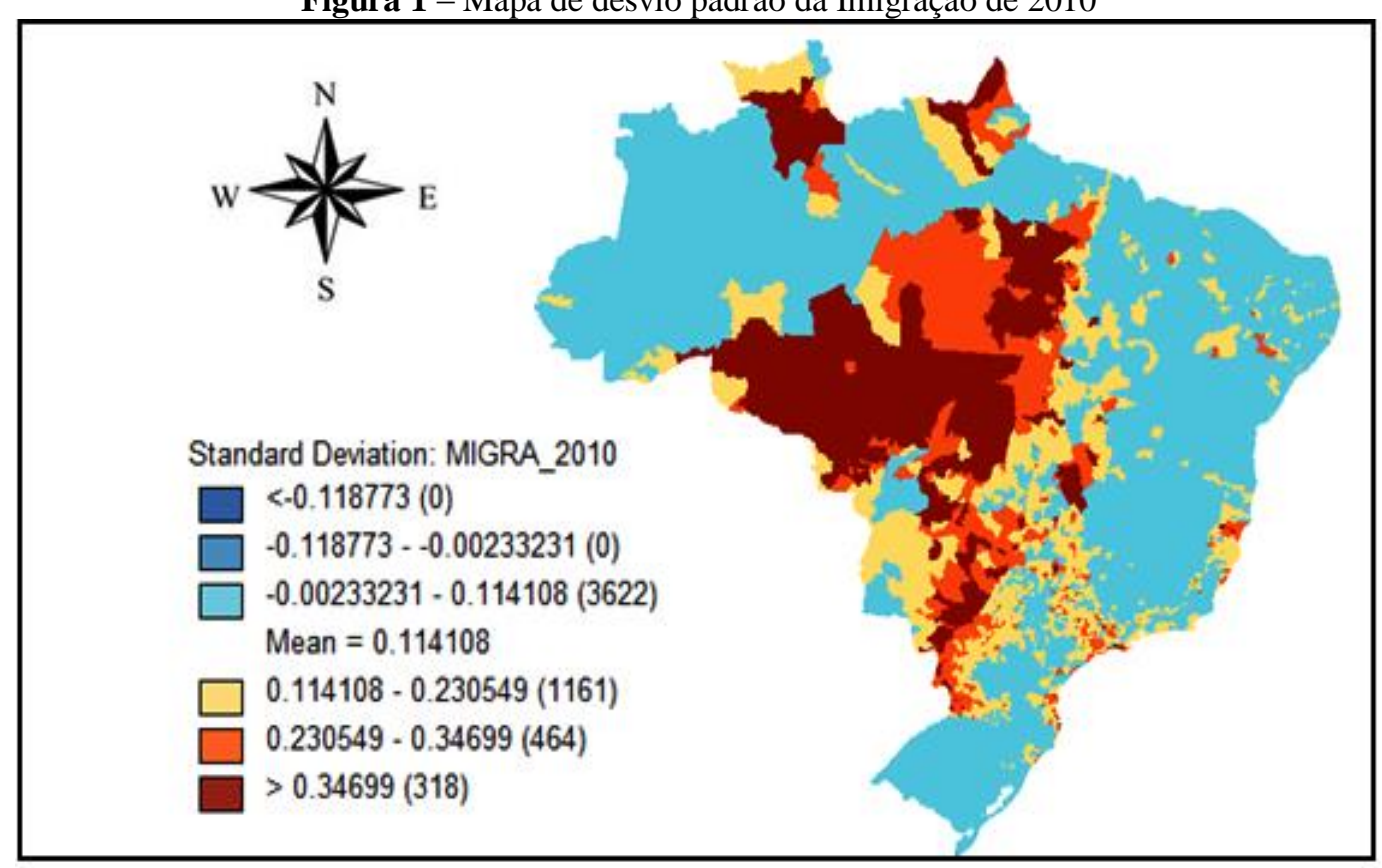

Fonte: Elaborado pelos autores com dados do IBGE e utilização do Sofware Geoda.

No ano de 2010, a imigração nacional apresentou em sua maioria uma taxa de imigração média, possuindo algumas regiões específicas uma taxa superior, como é o caso do Centro-Oeste e alguns estados do Norte, parte do oeste do Paraná e Santa Catarina.

As regiões que apresentaram maiores taxas de imigração, possuíram neste período uma melhora econômica, o que justificar esta atração de pessoas. No Norte, o estado do Pará tem um potencial natural que pode ser intensificado que é o minério de ferro, que nos últimos anos vem recuperando valor. A região Centro-Oeste teve um crescimento econômico nos quatro estados, movido pelas atividades da indústria de transformação (cana-de-açúcar e aço) e comércio, destacando o varejista (IPECE, 2012). Já os estados do Sul, apresentaram uma saturação econômica nos grandes centros, fazendo com que a imigração se direcionasse a outras cidades nos estados.

A média da taxa de imigração para o ano de 2010 foi de 0,1141. Dos municípios brasileiros, $21 \%$ apresentaram taxa com um desvio padrão acima da média, já $8 \%$ apresentaram a taxa com dois desvios padrão acima da média e $6 \%$ foram considerados outliers, acima da média. As unidades municipais que apresentaram taxas com um desvio padrão abaixo da média representam $65 \%$ do total e nenhum município apresentou dois desvios padrão abaixo da média ou outliers abaixo da média. Isso pode ser explicado pelo

Econ. e Desenv., Santa Maria, vol. 27, n.1, p. 164 - 182, jan. - jul. 2015 
valor da média ser baixo e as taxas de imigração com dois desvios padrão e os outliers acima da média apresentarem valores negativos.

Na figura 2, verificam-se os valores de I de Moran para a variável imigração na análise univariada (a) e para as variáveis, imigração e rendimento médio para análise bivariada (b) na matriz de pesos espaciais do tipo Rainha (Queen). Como pode ser verificado, existe uma autocorrelação espacial positiva entre as variáveis, a um nível de significância de 5\%.

Entretanto, conforme Pimentel e Haddad (2004), a análise da estatística I de Moran a respeito da existência de regimes espaciais, não torna possível a visualização de onde estão esses regimes. Para isso será utilizado o diagrama de dispersão de Moran.

Figura 2 - Diagrama de Dispersão de Moran Univariado e Bivariado da Imigração e rendimento médio para o ano de 2010.

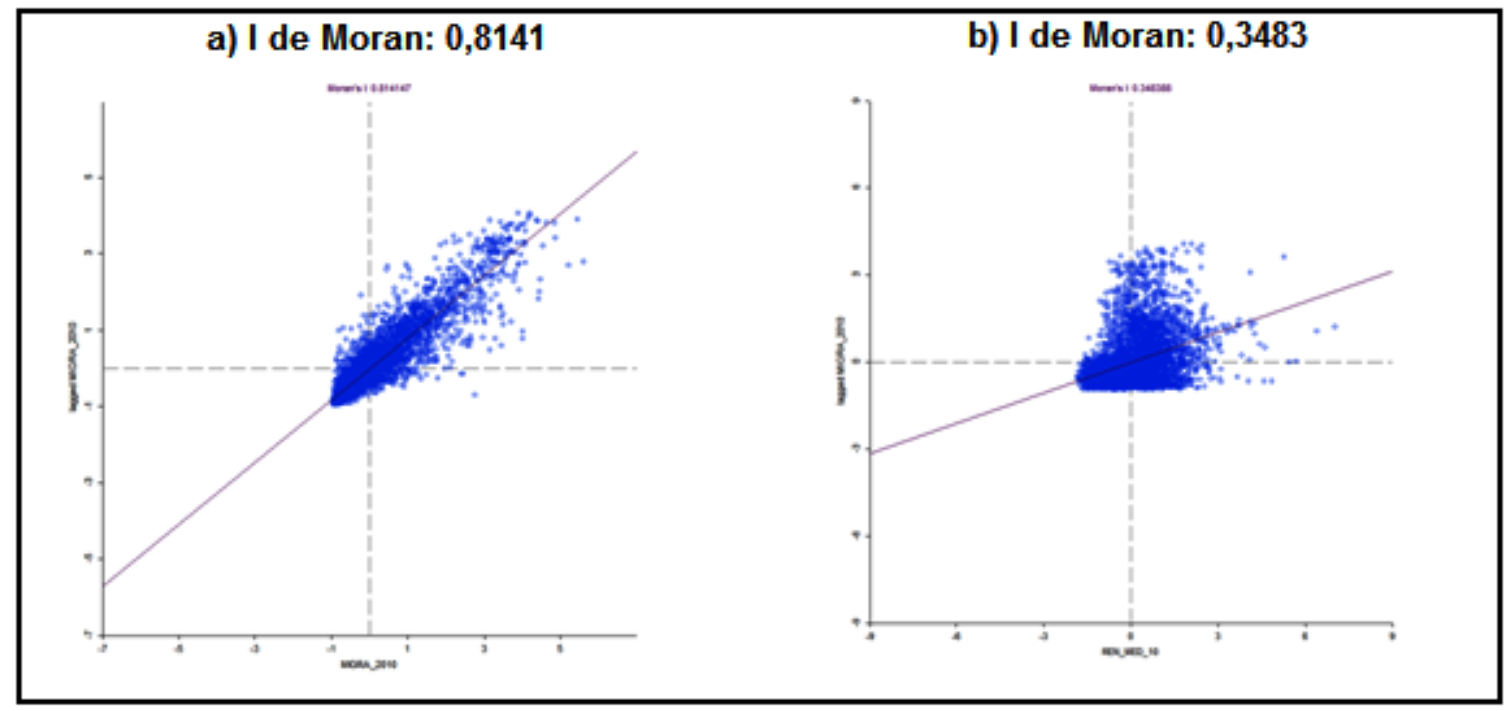

Fonte: Elaborado pelos autores com dados do IBGE e utilização do Software Geoda.

Conforme é apresentado na figura 2, verifica-se graficamente, no diagrama (a) a concentração espacial para imigração dos municípios brasileiros. Observando a variável, referente ao período de 2010, observa-se que 30\% dos municípios analisados encontram-se no quadrante AA e cerca de 59\% estão no quadrante BB. No que se refere às regiões atípicas, ou seja, aquelas que apresentam desvios em relação ao padrão global de autocorrelação se verificam que $6 \%$ dos municípios estão no quadrante BA e 5\% no quadrante AB. Há um menor número de municípios que recebem os migrantes e eles estão próximos a municípios que têm elevadas taxas de imigração e proporcionam maiores oportunidades para os migrantes conforme revela a figura $2 \mathrm{~b}$. Neste sentido, há maior variabilidade e dispersão dos dados no quadrante 1 , que corresponde ao quadrante A-A.

No diagrama (b) verifica-se a concentração espacial para imigração e rendimento médio dos municípios brasileiros. Observando as variáveis, referente ao ano de 2010, verifica-se que $27 \%$ dos municípios analisados encontram-se no quadrante AA e cerca de $44 \%$ estão no quadrante BB. No que se refere às regiões atípicas, ou seja, aquelas que apresentam desvios em relação ao padrão global de autocorrelação se verificam que $21 \%$ dos municípios estão no quadrante $\mathrm{BA}$ e $8 \%$ no quadrante $\mathrm{AB}$. Pode-se inferir dos resultados obtidos que há uma relação espacial forte positiva entre o crescimento dos rendimentos médios a intensidade da imigração. Regiões com baixos salários exibem baixa imigração, mas regiões com altos 
salários atraem imigrantes. Colocar quantos municípios estão em cada quadrante e o salário médio

Segundo Almeida (2004), é possível através de um mapeamento dos valores da probabilidade da medida, estatisticamente significativos, gerar um mapa de significância do Moran Local.

Nas figuras 3 e 4, os mapas de significância do ano 2010 apresentam que as regiões destacadas em verde escuro representam os regimes estatisticamente significativos a $0,1 \%$ e os municípios destacados em verde mais claro, as associações significativas a 5\%. Nos mapas de clusters do ano 2010, apresentam significância para o I de Moran local. De acordo com Almeida (2004), o mapa de cluster apresenta as quatro categorias estatisticamente significantes, combinando o mapa de significância local com o diagrama de dispersão de Moran.

Figura 3 - Mapa Lisa e de Cluster Univariado da imigração no ano de 2010.

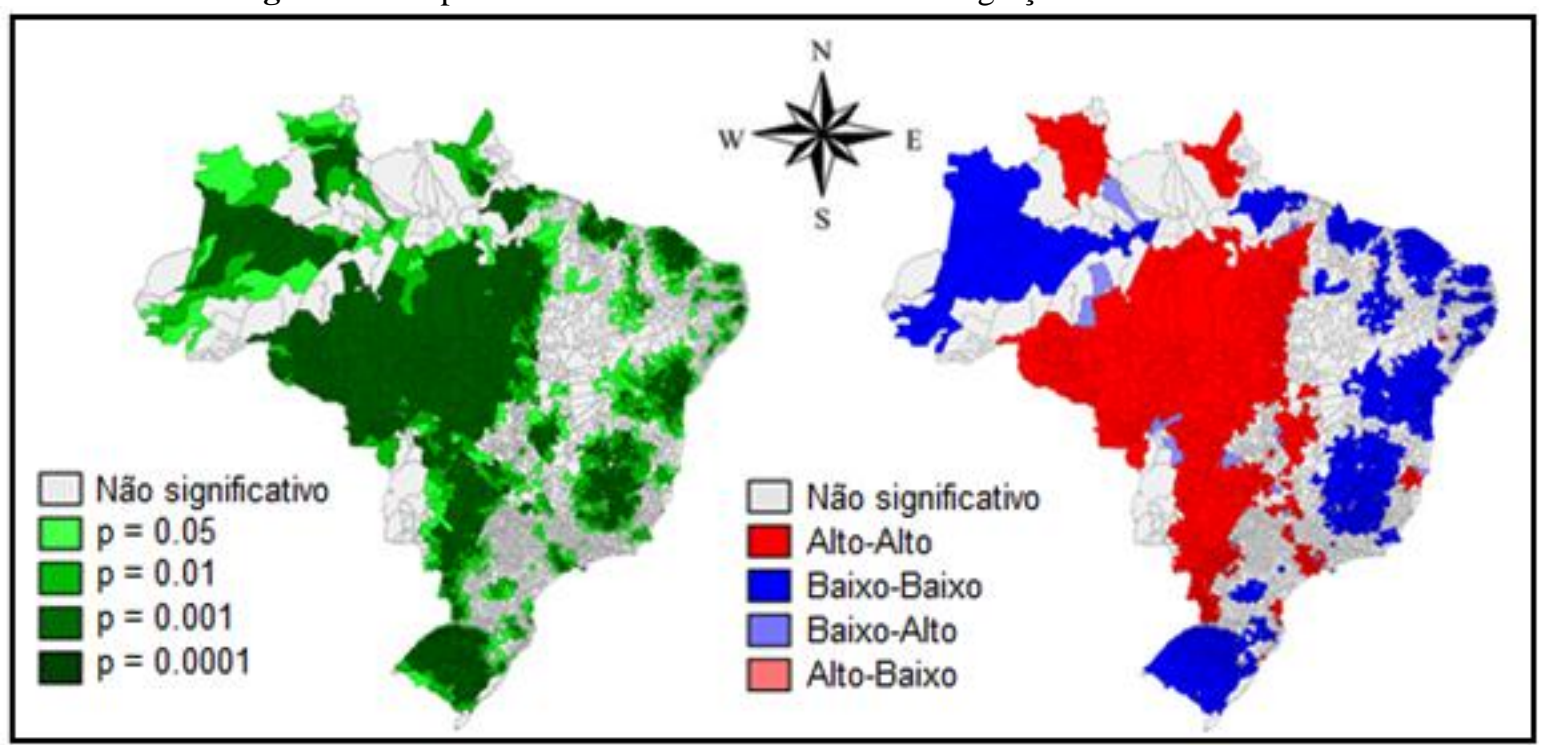

Fonte: Elaborado pelos autores com dados do IBGE e utilização do Sofware Geoda.

Na figura 3, são expostos os clusters de imigração, assim, os municípios responsáveis pela maior imigração nos estados apresentam alguma sinergia que resultam em externalidades espaciais positivas. Os clusters desta forma evidenciam uma tendência próxima do mapa de desvio padrão, onde as regiões que tiveram a maior concentração de relação espacial AltoAlto foram o Centro-Oeste, parte do Norte e parte do Oeste do Paraná e Santa Catarina.

Em relação aos estados que apresentaram menores taxas de imigração, são também os estados que apresentam historicamente o maior grau de eimigração, é importante destacar que o estado de Rio Grande do Sul e os estados nordestinos, estão apresentando um fenômeno parecido de eimigração, justificado pela melhora econômica das regiões (JARDIM; BARCELLO, 2011).

A Região Nordeste apresentou uma melhora significativa, devido às políticas econômicas regionais realizadas. Segundo Oliveira e Jannuzzi (2005), a região Nordeste apresenta estes movimentos migratórios que podem ser explicados por alguns fatores historicamente conhecidos como a estagnação econômica e níveis de desemprego nas áreas urbanas da região. Cunha e Baeninger (2000) relatam que as transformações na estrutura 
produtiva brasileira e as novas configurações do desenvolvimento regional, que se delineiam a partir da década de 1970, ambientam importantes modificações na dinâmica migratória nordestina. Os autores ainda enfatizam que o processo de desconcentração econômica, amparado pelas políticas de incentivo ao investimento industrial no Nordeste, influencia o comportamento da imigração nordestina na década de 80 , onde se destacam os fluxos de retorno.

Pode se observar no mapa de significância que $47 \%$ dos municípios do Brasil apresentaram significância de 5\% para as unidades espaciais da estatística I de Moran local para imigração no ano de 2010, sendo que $15 \%$ apresentaram significância de $0,1 \%$ e $29 \%$ significância de $1 \%$.

No mapa de cluster, verifica-se que no período de análise, o ano de 2010, dos municípios que foram significativos a 5\% (47\% dos municípios), as associações espaciais AA representaram 33\% dos municípios e se concentraram nas regiões Centro Oeste e Sul do país, as associações espaciais AA são regiões com alto percentual de imigração cercado por municípios que também apresentam alto percentual de imigração. O regime BB representa $66 \%$ dos municípios, concentrados no Norte, Nordeste e Sudeste do país e no Rio Grande do Sul, demonstrando aglomerações de municípios que apresentam baixo percentual de imigração cercados por municípios que apresentam a mesma característica. Por outro lado, as regiões atípicas (BA e $\mathrm{AB}$ ) são representadas por $1 \%$ dos municípios.

$\mathrm{Na}$ figura 4, é feito uma análise bivariada da imigração e renda per capita, esta análise teve o intuito de elucidar a relação entre imigração e renda exposta na literatura.

Figura 4 - Mapa Lisa e de Cluster Bivariado da imigração e renda per capita de 2010.

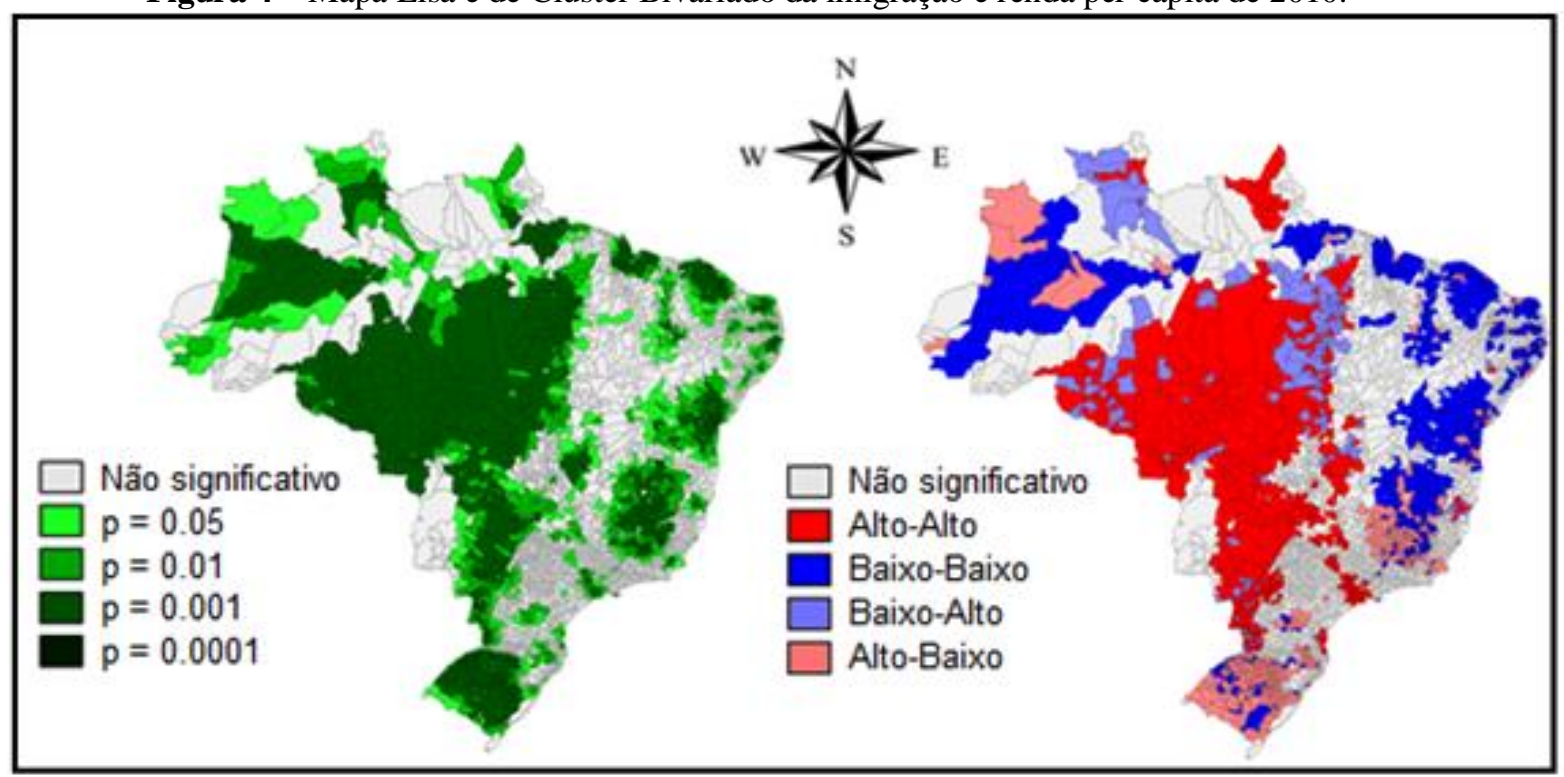

Fonte: Elaboração Própria com Dados do IBGE e Utilização do Software Geoda.

Desta forma, pode se observar no mapa de significância que também $47 \%$ dos municípios do Brasil apresentaram significância de 5\% para as unidades espaciais da estatística I de Moran local para rendimento médio e imigração no ano de 2010, sendo que $15 \%$ apresentaram significância de $0,1 \%$ e $29 \%$ significância de $1 \%$.

No mapa de cluster, pode se observar que no período de análise do ano de $2010,47 \%$ dos municípios foram significativos a $5 \%$. As associações espaciais AA representaram $27 \%$ RE\&D Econ. e Desenv., Santa Maria, vol. 27, n.1, p. 164 - 182, jan. - jul. 2015 
dos municípios e se concentraram nas regiões Centro Oeste e Sul do país, as associações espaciais AA são regiões que apresentam alta imigração com alto rendimento médio. $\mathrm{O}$ regime BB representa $46 \%$ dos municípios, concentrados nas regiões Norte, Nordeste e Sudeste do país, demonstrando aglomerações de municípios que apresentam baixa imigração com baixo rendimento médio. Por outro lado, as regiões atípicas (BA e AB) são representadas por 26\% dos municípios. Conforme destaca Souchad e Fusco(2012, p.15), os fenômenos migratórios recentes estão associados a movimentos de redistribuição populacional que favorecem a dinâmica do povoamento e a "integração e produção do espaço" e sinalizam a perspectiva de exploração de nichos de atividades.

\section{Considerações Finais}

Os resultados do presente estudo permitiram verificar o padrão locacional de imigração e a forte relação entre imigração e rendimento médio para os municípios dos brasileiros, verificando a autocorrelação espacial e a presença de clusters espaciais entre os municípios.

Através da Análise Exploratória de Dados Espaciais (AEDE) verificou-se na análise univariada da imigração, municípios com alta taxa de imigração estão cercados por municípios com alta taxa de imigração, assim como os municípios com baixa taxa de imigração estão cercados por municípios nas mesmas condições. Pela análise bivariada da imigração e do rendimento médio, municípios com alta taxa de imigração e alto rendimento médio estão cercados por municípios com a mesma característica, assim como os municípios que apresentam baixa taxa de imigração e baixo rendimento médio estão cercados por municípios na mesma situação.

Os resultados da AEDE apontam que a concentração de municípios com os maiores valores de imigração ocorre nas regiões Centro Oeste e Sul do Brasil, e a concentração de municípios com os menores valores de imigração estão no Norte, Nordeste e Sudeste do país e no Rio Grande do Sul. Também se verificou que a concentração de municípios com os maiores valores de imigração e rendimento médio estão também no Centro Oeste e Sul do Brasil, e a concentração de municípios com os menores valores de imigração e rendimento médio estão no Norte, Nordeste e Sudeste do país.

\section{Referências}

ALMEIDA, E. Econometria espacial aplicada. Campinas, SP. Ed. Alínea, 2012.

ALMEIDA, E. S. Curso de econometria espacial aplicada. Piracicaba: ESALQ, 2004. (Material Didático)

ANSELIN, L. Spatial econometrics: methods and models. Boston: kluwer Academic, 1998.

AZZONI, C.; CARMO, H.; MENEZES, T. Comparação da paridade do poder de compra entre cidades: aspectos metodológicos e aplicação ao caso brasileiro. Pesquisa e

planejamento econômico, Rio de Janeiro, v. 33, n. 1, p. 91-126, abr. 2003.

Econ. e Desenv., Santa Maria, vol. 27, n.1, p. 164 - 182, jan. - jul. 2015 
BORJAS, G. J. Economic theory and international migration. International Migration Eview. v. 23, n. 3, Special Silver Anniversary Issue: International Migration an Assessment for the 90's, autumn/1989, p. 457-485, 1989.

BRITO, F. As migrações internas no Brasil: um ensaio sobre os desafios teóricos recentes. Belo Horizonte: UFMG/Cedeplar, 2009. 20. Disponível em www.abep.nepo.unicamp.br/docs/anais/outros/6EncNacSobreMigracoes/ST3/FaustoBrito.pdf

CAMARA, M. R. G.. Migrações internas: evolução teórica e algumas evidências empíricas para o caso brasileiro. In: Semina: Ci. Soc./Hum. Londrina, v.9, n.2, p.110-116, 1988.

COSTA, M. Estudos de demografia urbana. Rio de Janeiro, IPEAI-INPES, 1975.

CUNHA. J.M.; BAENINGER, R. A imigração nos estados brasileiros, no período recente: principais tendências e mudança. In: ENCONTRO NACIONAL SOBRE IMIGRAÇÃO, 2 ., Anais... Belo Horizonte: Abep, 2000. p. 117-165.

DURHAM, E.. A caminho da cidade. Ed. Perspectiva, 1973.

FREGUGLIA, R. S.; PROCOPIO, T. S. Efeitos da mudança de emprego e da imigração interestadual sobre os salários no Brasil Formal: Evidencia a partir de dados em Painel. Pesquisa e planejamento econômico, PPE, v. 43, n. 2, ago. 2013.

GREENWOOD, J. M.; HUNT, G.L. The early history of migration research. International Regional Science Review, 26,1,p. 3-37, 2003.

GOLGHER, A. B.; ROSA, C. H.; ARAÚJO JÚNIOR, A. F. The determinants of migration in Brazil. (Texto para Discussão, n. 268). UFMG/Cedeplar, Jul. 2005.

GRAHAM, D. H.; HOLANDA FILHO, S. B. Interregional and urban migration and economic growth in Brazil. In: Migrações internas e desenvolvimento regional.

CEDEPLAR/UFMG, Mimeo, 1973.

IBGE, Censo Demográfico 2010. Acesso em 04 de dezembro e 2014. Disponível em http://www.ibge.gov.br/home/presidencia/noticias/imprensa/ppts/00000008473104122012315 727483985.pdf.

IPECE - INSTITUTO DE PESQUISA E ESTRATÉGIA ECONÔMICA DO CEARÁ. IPECE Informe, no 46, Novembro de 2012. Disponível em: www ipece.ce.gov.br/ publicacoes/ipece-informe/Ipece_Informe_46_28_novembro 2012.pdf. Acessado em: $10 \mathrm{de}$ abril de 2015.

JARDIM, M. L.; BARCELLO, T. M. M.. Migrações no Rio Grande do Sul. Revista Paranaense de Desenvolvimento, Curitiba, n.121, p.133-147, jul./dez. 2011.

JUSTO, W. R.; SILVEIRA NETO, R. M. Quem são e para onde vão os migrantes no Brasil? O perfil do migrante interno brasileiro. In: Anais do XXXVI Encontro Nacional de Economia. Salvador : Anpec, 2008. 
JUSTO, W. R.; FERREIRA, R. A.; LIMA, F.; MARTINS, G. N.. Imigração Intermunicipal no Brasil: A dinâmica dos fluxos migratórios municipais. Revista Economia e

Desenvolvimento, n. 21, p. 108-129, 2009.

LANGONI, C. G.. Distribuição da renda e desenvolvimento econômico do Brasil. Rio de Janeiro, Editora Expressão e Cultura, 1973.

LEE, E. S. Theory on migration. Demography, v. 3, nº 1, p.47-57, 1966.

Lima, R. Mercado de trabalho: O capital humano e a teoria da segmentação. Pesquisa Planejamento Econômico, 1980.

MATA, M. Urbanização e migrações internas. Pesquisa e planejamento econômico, 1973.

MATA, M.; CARVALHO, E. W. R.; SILVA, M. T. L. L. C. Migrações internas no Brasil: aspectos econômicos e demográficos. IPEA/INPES, Rio de Janeiro, 1973.

MENEZES, T. A.; FERREIRA, J. D. Imigração e convergência de renda. São Paulo: USP/NEREUS, (Texto de Discussão, n. 13), 2003.

MATA, M. Urbanização e migrações internas. In: MOURA, H. (org.). Imigração interna; textos selecionados. Fortaleza: BNB, 1980.

MINCER, J. Investment in human capital and personal income distribution. Journal of Political Economy, n66, p. 281-302, 1958.

OLIVEIRA, K. F.; JANNUZZI, P. M.. Motivos para imigração no Brasil e retorno ao Nordeste: padrões etários, por sexo e origem/destino. São Paulo Perspec. vol.19, nº 4, São Paulo Oct./Dec, 2005. Disponível em: www.scielo.br/ pdf/spp/v19n4/ v19n4a09.pdf. Acessado em: 11 de marco de 2015.

OLIVEIRA, K. F.; JANNUZZI, P. M. Motivos para imigração no Brasil: padrões etários, por sexo e origem/destino. In: ENCONTRO NACIONAL DE ESTUDOS POPULACIONAIS, XIV. Anais, 2004.

PIMENTAL, E.A; HADDAD, E.A. Análise da distribuição espacial da renda no estado de Minas Gerais: uma abordagem setorial. 2004. Disponível em: http://www.econ.fea.usp.br/ nereus_02_04.pdf. Acesso em 20 set. 2014.

QUEIROZ, S. N.; BAENINGER, R.. Imigração de Retorno: O Caso Recente das Migrações Cearenses. Rev. Econ. NE, Fortaleza, v. 44, n. 4, p. 833-850, out-dez, 2013. Disponível em: http://www.bnb.gov.br/projwebren/Exec/artigoRenPDF.aspx?cd_artigo_ren=1400. Acessado em: 11 de março de 2015.

RAVENSTEIN, E. G.. The laws of migration. Journal of the Royal Statistical Society, Vol. 48, Part II, p. 167-227, 1885.

ROCHA, C. B. ; PARRÉ, J. L. . Estudo da distribuição espacial do setor agropecuário do Rio Grande do Sul. In: VI Encontro Nacional da Associação Brasileira de Estudos Regionais e 
Urbanos, 2008, Aracaju. Anais do ... Encontro Nacional da Associação Brasileira de Estudos Regionais e Urbanos, 2008.

SACHSIDA, A; CASTRO, P. F.; MENDONÇA, M. J. C.; ALBUQUERQUE, P. H. Perfil do migrante brasileiro. (Texto para Discussão), n. 1410. Rio de Janeiro: IPEA, 2009.

SACHSIDA, A.; CAETANO, M.; ALBUQUERQUE, P. Distribuição de renda, transferências federais e imigração: um estudo de dados de painel para as Unidades da Federação do Brasil. (Texto para Discussão, n. 1471). Brasília: 2010.

SANTOS, M. A.; BARBIERI, A. F.; CARVALHO, J. A. M.; MACHADO, C. J. . Imigração: Uma revisão sobre algumas das principais teorias. Texto para discussão $\mathbf{n}^{\mathbf{0}} \mathbf{3 9 8}$, CEDEPLAR, 2010.

SJAASTAD, L. A. The Costs and Returns of Human Migration. Journal of Political Economy, v. 70, n. 5, Part 2: Investment in Human Beings (Oct., 1962), p. 80-93.

SINGER, P. "Migrações internas: considerações teóricas sobre o seu estudo", in Economia política da urbanização. São Paulo: Editora Brasiliense/CEBRAP, p. 29-60, 1973.

SOUCHAUD, S.; FUSCO, W. População e ocupação do espaço: o papel das migrações no Brasil. Redes, v. 17, n. 2, p. 5-17, 2012.

TAYLOR, J. E.. Differential Migration, Networks, Information and Risks. In: STARK, O. (volume editor); Migration, Human Capital and Development. Greenwich, Connecticut, Jai Press Inc., v. 4, 1986.

TAYLOR, J. E. International migration, remittances and development: myths and facts. International Migration, v.37, n.1, p.63-88, 1999.

TONET, F. M.; Imigração Interna e Seletividade: uma aplicação para o Brasil. In: $39^{\circ}$ Encontro Nacional de Economia, 2011, Foz do Iguaçu. Anais do $3^{\circ}$ Encontro Nacional de Economia - ANPEC, 2011. 